\title{
Meissner effect in a bosonic ladder
}

\author{
E. Orignac \\ LPTENS CNRS UMR 8549 24, Rue Lhomond 75231 Paris Cedex 05, France
}

\author{
T. Giamarchif \\ Laboratoire de Physique des Solides, CNRS-UMR 8502, UPS Bât. 510, 91405 Orsay France
}

(Dated: September 1, 2018)

\begin{abstract}
We investigate the effect of a magnetic field on a bosonic ladder. We show that such a system leads to the one dimensional equivalent of a vortex lattice in a superconductor. We investigate the physical properties of the vortex phase, such as vortex density and vortex correlation functions and show that magnetization has plateaus for some commensurate values of the magnetic field. The lowest plateau corresponds to a true Meissner to vortex transition at a critical field $H_{c 1}$ that exists although the system has no long range superconducting order. Implications for experimental realizations such as Josephson junction arrays are discussed.

PACS numbers: 05.30.Jp, 71.10.Pm, 74.50.+r
\end{abstract}

The effect of a magnetic field on interacting particles is a long standing problem. A spectacular case is provided by type II superconductor, in which the magnetic field is totally expelled below $H_{c_{1}}$, whereas a vortex state exists for $H>H_{c_{1}}$. This behavior, however, is obtained from the Landau-Ginzburg equation, and it is important to know what happens when interactions and fluctuations have more drastic effects, such as in one dimensional systems. Indeed, in a one dimensional conductor at $T=0$, although there is no long range order, superconductivity in the sense of infinite d.c. conductivity can nevertheless be presentll. For a one dimensional chain, there is no orbital effect, and this question is not relevant. However, a system made of a finite number of coupled chains (a ladder), is still one dimensional (no long range order can exist) but orbital effect of the magnetic field is present, opening the possibility of such a transition.

Beyond its own theoretical interest the investigation of the effect of a magnetic field on ladder systems is also of direct experimental relerance, due to the various realizations of such ladders 2 月. superconducting both from attractive (s-wave) and repulsive interactions (d-wave). In the attractive case, the system is close to standard superconductors where pairs of fermions can hop from one chain to the other leading to a Josephson coupling, provided the applied magnetic field is smaller that the spin gap. The system can thus be desfribed as a bosonic ladder. Josephson junction arrays 5 brovide also a very direct realization of such a bosonic ladder 0 and are thus the prime candidates to observe these effects. This problem of Josephson ladders has been investigated previous y in the classicall and quantum limit both analytically 20.11 and numerically 12 in the high field limit of half a flux quantum per plaquette for one dimensional situations For the Josephson two leg ladder, it has been shown 1011 that a true transition exists between a commensurate and a vortex phase, however the detailed behavior of the vortex phase and the effects of commensurability of the magnetic field remain to be understood.
In this paper we investigate the effect of a magnetic field directly on the bosonic two leg ladder. We study the Meissner-vortex transition in this system, and the nature of the vortex phase. We show that plateaus in the magnetization for commensurate values of the field exist, in a way similar to the Mott transition in one dimension for commensurate values of the filing. We analyse the consequences for transport in such bosonic ladders.

The lattice Hamiltonian of the bosonic two leg ladder in a magnetic field is:

$$
\begin{aligned}
H & =-t_{\|} \sum_{i, p=1,2}\left(b_{i+1, p}^{\dagger} \mathrm{e}^{i e^{*} a A_{\|, p}(i)} b_{i, p}+b_{i, p}^{\dagger} \mathrm{e}^{-i e^{*} a A_{\|, p}(i)} b_{i+1, p}\right) \\
& -t_{\perp} \sum_{i}\left(b_{i, 2}^{\dagger} e^{i e^{*} A_{\perp}(i)} b_{i, 1}+b_{i, 1}^{\dagger} e^{-i e^{*} A_{\perp}(i)} b_{i, 2}\right) \\
& +U \sum_{i, p} n_{i, p}\left(n_{i, p}-1\right)+V n_{i, 1} n_{i, 2}
\end{aligned}
$$

where the density $n_{i, p}=b_{i, p}^{\dagger} b_{i, p}$ and the magnetic field is introduced via the Peierls substitution. In addition to their parallel $t_{\|}$and perpendicular $t_{\perp}$ hopping the bosons repel via an on-site $U$ and an inter-chain $V$ repulsion. The presence of a uniform magnetic field implies that:

$$
\oint \mathbf{A} \cdot d \mathbf{l}=A_{\perp}(i+1)+a A_{\|, 1}(i)-A_{\perp}(i)-a A_{\|, 2}(i)=\Phi
$$

Where $\Phi$ is the flux of the magnetic field through a plaquette. The low energy properties of (11) are more transparent 13 using a "bosonized" representation of the boson operators 14 . One obtains:

$$
\begin{array}{r}
H=\sum_{p=1,2} \int \frac{d x}{2 \pi}\left[u K\left(\pi \Pi_{p}-e^{*} A_{\|, p}\right)^{2}+\frac{u}{K}\left(\partial_{x} \phi_{p}\right)^{2}\right] \\
-\frac{t_{\perp}}{\pi a} \int d x \cos \left(\theta_{1}-\theta_{2}+e^{*} A_{\perp}(x)\right)+ \\
\frac{V a}{\pi^{2}} \int d x \partial_{x} \phi_{1} \partial_{x} \phi_{2}+\frac{2 V a}{(2 \pi a)^{2}} \int d x \cos \left(2 \phi_{1}-2 \phi_{2}\right)
\end{array}
$$


where $\phi_{i}$ and $\pi \Pi_{i}=\nabla_{x} \theta_{i}$ are conjugate variables and the boson annihilation operator is given by:

$$
\psi_{p}(x=n a)=\frac{b_{n}}{\sqrt{a}}=\frac{e^{i \theta_{p}(x)}}{\sqrt{2 \pi a}}
$$

where $a$ is the lattice spacing along the legs of the ladder. $u$ is the sound velocity of the collective density oscillations. The linear nature of the spectrum is the signature that true superfluidity exist in a single chain even without long range ordert. $K$ is the Luttinger parameter, directly related to the compressibility of the system, and incorporating all microscopic interaction effects. For a simple onsite repulsion $1<K<\infty, K=\infty$ being free bosons and $K=1$ hard core bosons. For more general (longer range) interactions all values of $K$ are in principle allowed. If one introduces the symmetric and antisymmetric combinations $\phi_{s, a}=\frac{\phi_{1} \pm \phi_{2}}{\sqrt{2}}$ (and similar combinations for $\Pi$ and $A$ ), Eq. (3) reads $\$ 3$ :

$$
\begin{aligned}
H & =H_{s}^{0}+H_{a}^{0}-\frac{t_{\perp}}{\pi a} \int d x \cos \left(\sqrt{2} \theta_{a}+e^{*} A_{\perp}(x)\right) \\
& +\frac{2 V a}{(2 \pi a)^{2}} \int d x \cos \sqrt{8} \phi_{a}
\end{aligned}
$$

where $(\nu=s, a)$ :

$$
H_{\nu}^{0}=\int \frac{d x}{2 \pi}\left[u_{\nu} K_{\nu}\left(\pi \Pi_{\nu}-e^{*} A_{\nu}\right)^{2}+\frac{u_{\nu}}{K_{\nu}}\left(\partial_{x} \phi_{\nu}\right)^{2}\right]
$$

whith $K_{s}=K\left(1+\frac{V K a}{\pi u}\right)^{-1 / 2}, K_{a}=K\left(1-\frac{V K a}{\pi u}\right)^{-1 / 2}$. $A_{s}$ describes an Ahronov-Bohm Flux threading the system. In the following, we assume that there is no such flux, so $A_{s}=0$. We also assume that $K_{a}>1$, so that the term $\cos \sqrt{8} \phi_{a}$ is irrelevant. This leads to an Hamiltopian similar to the one derived for the Josephson ladder 10.11 . The total and antisymmetric longitudinal current $j_{s, a}=j_{1} \pm j_{2}$, and the current perpendicular to the ladder are given by $(\nu=s, a)$ :

$$
\begin{aligned}
& j_{\nu}=u K e^{*} \sqrt{2}\left(\Pi_{\nu}-\frac{e^{*}}{\pi} A_{\nu}\right) \\
& j_{\perp}=\frac{e^{*} t_{\perp}}{\pi a} \sin \left(\sqrt{2} \theta_{a}+e^{*} A_{\perp}\right)
\end{aligned}
$$

$H_{s}$ gives a simple dynamics, the behavior of $H_{a}$ is richer. In the absence of a magnetic field, $H_{a}$ is a sineGordon Hamiltonian. For $K>1 / 4$, it develops an order in the field $\theta_{a}$ with $\left\langle\theta_{a}\right\rangle=0$ and a gap in the excitation spectrum $\Delta_{a} \sim \frac{u}{a}\left(\frac{t_{\perp} a}{u}\right)^{\frac{1}{2-1 /(2 K)}}$. This describes the locking of the relative phases by Josephson coupling. Let us now consider the effect of a magnetic field such that the flux per plaquette $\Phi=\frac{2 \pi}{e^{*}} \frac{p}{q}$, where $p, q$ are two mutually prime integers. Using the gauge $A_{a}=0, A_{\perp}=\Phi \frac{x}{a}$, we can write:

$$
\begin{aligned}
H_{a} & =\int \frac{d x}{2 \pi}\left[u_{a} K_{a}\left(\pi \Pi_{a}\right)^{2}+\frac{u_{a}}{K_{a}}\left(\partial_{x} \phi_{a}\right)^{2}\right] \\
& -\frac{t_{\perp}}{\pi a} \int d x \cos \left(\sqrt{2} \theta_{a}+\frac{2 \pi p}{q} \frac{x}{a}\right)
\end{aligned}
$$

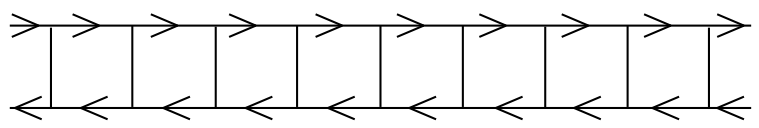

(a)

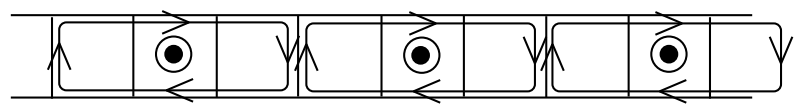

(b)

FIG. 1: The phases of the bosonic two leg ladder in the presence of a magnetic field. (a) The low field phases. Current only exist along the legs of the ladder leading to screening of the applied magnetic field. (b) the high field phase. Current exist both on the legs and the rungs of the ladder leading to a vortex lattice (here shown with a vortex every three sites). At commensurable flux the vortex lattice is perfectly ordered (at $T=0$ ) whereas away from commensurability the vortex system has only quasi long range positional order and no perfect crystalline order.

The Hamiltonian (9) is similar to the one describing the Mott transition in one dimension15, where any commensurability can in principle give a transition depending on $K$. Indeed, even if the presence of an oscillating phase in (9) makes $t_{\perp}$ naively_iprelevant, perturbation theory to order $q$ in $\frac{t_{\perp} a}{\pi u_{a}}$ shows 16.17 that a term:

$$
H_{q}=\frac{g_{q}}{\pi a} \int d x \cos q \sqrt{2} \theta_{a}
$$

exists with $g_{q} a /\left(\pi u_{a}\right) \sim\left(t_{\perp} a / \pi u_{a}\right)^{q}$ in perturbation. More generally this term is allowed by symmetry and is present in the Hamiltonian even beyond perturbation theory. The term (10) opens a gap $\Delta_{p / q}=$ $\frac{\pi u}{a}\left(\frac{t_{\perp} a}{\pi u_{a}}\right)^{\frac{2 K_{a} q}{4 K_{a}-q^{2}}}$ provided $K_{a}>\frac{q^{2}}{4}$. The expectation value of $\theta_{a}$ in the gapped phase is $\left\langle\theta_{a}\right\rangle=(2 k+1) \pi /(q \sqrt{2})$ where $k=0, \ldots, q-1$. This corresponds to a $q$-fold degenerate ground state resulting from the breaking of the discrete translation symmetry in direct analogy with Mott systems 15 or spin systems 18 . 19 . The corresponding expectation value of $j_{\perp}(x)$ is:

$$
\left\langle j_{\perp}(x)\right\rangle \propto \frac{e^{*} t_{\perp}}{\pi a} \sin \left[\frac{(2 k+1) \pi}{q}+\frac{2 \pi p}{a} \frac{x}{a}\right]
$$

giving a periodic pattern of the transverse currents of period $q a$ as shown in Fig. 11. This pattern corresponds to a vortex lattice phase pinned to the microscopic lattice with $p$ vortices in a supercell of $q$ sites, leading to an average vortex density $\bar{\rho}_{V}=\frac{p}{q}$. Let us now take a flux per plaquette which is an irrational multiple of the quantum of flux. If this value is close to a rational number with a small enough denominatorwe can decompose $e^{*} \Phi=$ $2 \pi \frac{p}{q}+\delta \Phi$. The most convenient gauge choice is then $A_{\perp}=\left(2 \pi e^{*} p\right) / q$ and $A_{a}=(\delta \Phi) /(a \sqrt{2})$. Introducing the 
field $P_{a}$ conjugate to $\theta_{a}, \pi P_{a}=\partial_{x} \phi, H_{a}$ becomes:

$$
\begin{aligned}
H_{a} & =\int \frac{d x}{2 \pi}\left[\frac{u}{K}\left(\pi P_{a}\right)^{2}+\frac{u}{K}\left(\partial_{x} \theta_{a}+\frac{e^{*} \Phi}{a \sqrt{2}}\right)^{2}\right] \\
& -\frac{g_{q}}{\pi a} \int d x \cos q \sqrt{2} \theta_{a}
\end{aligned}
$$

The deviation of the flux per plaquette from a rational value in the unit of the quantum of Hyx causes a commensurate-incommensurate transition 20.21.22: At low deviation, the system is in its commensurate phase, $\sqrt{2}\left\langle\theta_{a}\right\rangle=(2 k+1) \pi / q$. This implies $\left\langle j_{a}\right\rangle=-u K \frac{\left(e^{*}\right)^{2} \delta \Phi}{\pi a}$ and no modification of the perpendicular current $\left\langle\delta j_{\perp}\right\rangle=$ 0 and $\left\langle\delta j_{\perp}(x) \delta j_{\perp}\left(x^{\prime}\right)\right\rangle \sim e^{-\left|x-x^{\prime}\right| / \xi_{a}}$ with $\xi_{a}=u / \Delta_{a}$. In particular, for low magnetic field $(p=0, q=1)$, there is a Meissner current circulating only on the edges of the system (see-Fig. 1) and proportional to the applied vector potential10. For a large enough deviation from rationality, $\frac{u}{a} \delta \Phi>\Delta_{p / q}$, there is a transition to a phase where the $\cos q \sqrt{2} \theta_{a}$ operator is irrelevant. In this phase $\left\langle\partial_{x} \theta_{a}\right\rangle /(\pi \sqrt{2})$ is non zero an defines an order parameter. In the small applied field case 10 , this order parameter is just the vortex density $\rho_{V}$. In the general case, this order parameter measures the deviation $\delta \rho_{V}=\rho_{V}-\frac{p}{q}$ of the vortex density from a rational value. The average transverse current is zero in this phase. However, transverse currents correlations have an oscillating component that decays with a power law as

$$
\left\langle j_{\perp}(x) j_{\perp}\left(x^{\prime}\right)\right\rangle \sim \frac{\cos \left[2 \pi \bar{\rho}_{V}\left(x-x^{\prime}\right)\right]}{\left|x-x^{\prime}\right|^{1 / K_{a}^{*}}}
$$

where $K_{a}^{*}$ is the renormalized Luttinger parameter. It would be worthwhile to check in a numerical simulation such as 12 whether such current correlations are present. There is a pattern of transverse currents alternating along the rungs of the ladder. One can identify the solitons in $\theta_{a}$ with vortices surrounded by circulating currents (see figure 11. If a vortex is at position $x$, it causes a jump of $2 \pi$ in $\theta_{1}-\theta_{2}$ at this point. This enables us - oo write the density of these one-dimensional objects as 14 :

$$
\rho_{V}(x)=\frac{1}{\pi \sqrt{2}} \partial_{x} \theta_{a}+\sum_{m=-\infty}^{m=\infty} \frac{C_{m}}{\pi a} e^{i m\left[2 \pi \bar{\rho}_{V} x+\sqrt{2}\left(\theta_{a}-\left\langle\theta_{a}\right\rangle\right)\right]}
$$

When the density of vortices is $p / q$, the potential energy term (10) can be interpreted as the coupling of the vortex density to a pinning potential of period $q$ lattice spacings. Therefore we recover the interpretation in term of pinning of the vortices by the underlying microsfopic lattice and the analogy with the Mott transition 1 is in $d=1$. Eq. (14) gives as vortex current $j_{V}(x)=-\frac{\partial_{t} \theta_{a}}{\pi \sqrt{2}}$ The application of a voltage $V_{1}-V_{2}$ between the chains gives: $\left\langle\partial_{x} \phi_{a}\right\rangle=e^{*} \frac{V_{1}-V_{2}}{u_{a} K_{a} \sqrt{2}}$ and $V_{1}-V_{2}=E_{\perp} b=-\frac{2 \pi}{e^{*}} j_{V}$, which is just Faraday's law. This completes the identification of the incommensurate phase as an unpinned vortex lattice. Because of the linearization of the spectrum in (3) there

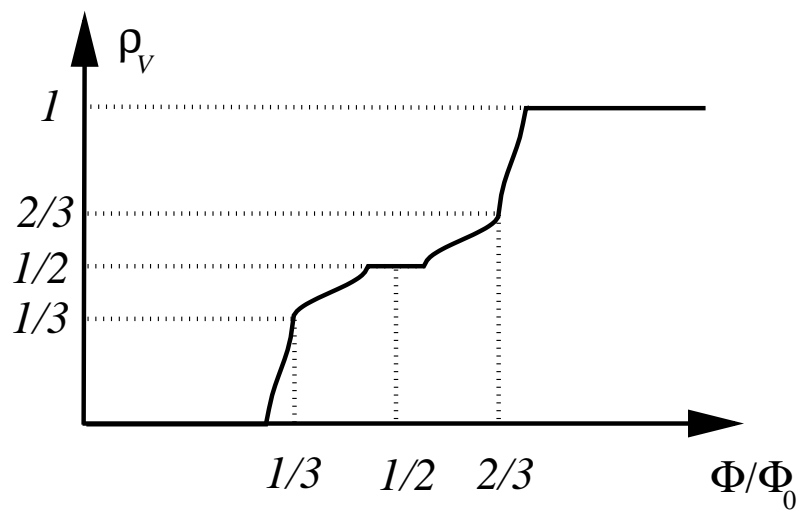

FIG. 2: Sketch of the staircase in the magnetization of the bosonic or Josephson ladder. On the figure, $K_{a}=4$, so that only the plateaus obtained for $p / q=0,1 / 3,1 / 2,2 / 3,1$ survive to the quantum fluctuations. Note that the width of the $1 / 3$ and $2 / 3$ plateaus is already extremely reduced compared with the width of the $1 / 2$ plateau.

is no Laplace force on vortices. This pathology could be cured by putting back the band curvature.

In the classical case 1 , a vortex lattice phase is obtained each time the flux per plaquette in a rational multiple of the quantum of flux, leading to a devil's staircase structure in the behavior of the magnetization. Here, the quantum fluctuations wipe out the large fractions for which $q^{2}>4 K_{a}$, so only some plateaux remain as shown on Fig. 2. As quantum fluctuations are reduced, more and more plateaus in the magnetization curve are formed. It is interesting to study how the width of a magnetization plateau increases as a function of $K_{a}$ or $t_{\perp}$. Standard RG calculation of the gap show that the width of a plateau behaves as $\exp \left(-C / \sqrt{t_{\perp}-t_{\perp}^{c}}\right)$ close to the threshold. For $\Phi=\frac{\pi}{e^{*}}$, a vortex lattice phase has been obtained in numerical simulations 12 . It remains to be seen whether such phase is a Luttinger Liquid or a pinned vortex lattice.

Close to the transition the coefficient $K_{a}^{*}$ is universal and $K_{a}^{*}=q^{2} / 2$. It is pessible in the limit $\delta \Phi \rightarrow$ $\delta \Phi_{c 1}(p / q)$ to refermionize 202 yielding an average vortex density $\left\langle\rho_{V}\right\rangle=\frac{e^{*}}{2 \pi a} \sqrt{\Phi^{2}-\Phi_{c}^{2}}$ and vortex current $\left\langle j_{a}\right\rangle=\frac{u\left(e^{*}\right)^{2}}{2 \pi a}\left(\sqrt{\Phi^{2}-\Phi_{c}^{2}}-\Phi\right)$. The transition from the pinned vortex lattice state to the vortex liquid state is thus continuous. The behavior of the average current as a function of flux is plotted on figure 3. This is reminiscent of the behavior of a Type II superconductor, however the growth of the magnetization is here quite different 10 from the standard $B=H+M \sim \frac{1}{\ln ^{2}\left(H-H_{c 1}\right)}$ law.

How could the Meissner and the vortex lattice phase be detected in an experiment? First, $h_{c_{1}}=\frac{\Phi_{c}}{a b}$ must be smaller than the spin gap. Otherwise, the magnetic field would break the Cooper pairs before vortices are nucleated, a situation reminiscent of type I superconductivity. Second, in a standard material, $a \simeq 1$ Åso that $h_{c_{1}} \sim$ $\frac{h}{2 e^{*} a^{2}} \simeq 2.1 \times 10^{5} \mathrm{~T}$ making the vortex lattice unobserv- 


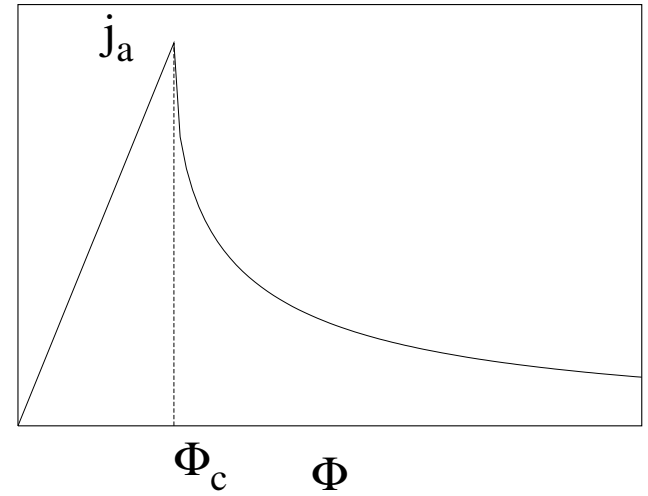

FIG. 3: The behavior of the screening current with $\Phi$. For $\Phi<\Phi_{c}$, the system is in the Meissner phase and the screening current increases linearly with $\Phi$. For $\Phi>\Phi_{c}$, the screening current decreases with the applied field.

able. For Josephson junction ladders, $a \simeq 1 \mu \mathrm{m}$, giving a typical $h_{c 1} \simeq 2.1 \times 10^{-3} \mathrm{~T}$ which is easily achieved. In principle, the formation of the vortex lattice in this system could be observed by magnetization measurements or by resolving individual vortices as was done for other mesoscopic systems 23. The Meissner transition also affects transport properties at a commensurate filling of an integer number of Cooper pairs per island. In this case, a $\cos 2 \phi_{s} \cos 2 \phi_{a}$ term is present and favors an insulat- ing state1. The interchain Josephson coupling $\cos \sqrt{2} \theta_{a}$ competes with this term making the system conducting when large enough 24. The application of $H>H_{c 1}$ suppresses the interchain Josephson coupling turning the ladder insulating in the vortex phase. The Meissner transition is thus accompanied by a superconductor-insulator transition in this case. At incommensurate filling, the magnetic field still affects transport properties if there is an artificial defect in the ladder, which adds a term $V_{1} \cos \sqrt{2} \phi_{s}(x=0) \cos \sqrt{2} \phi_{a}(x=0)+V_{2} \cos \sqrt{8} \phi_{s}(x=0)$ to the Hamiltonian. In the Meissner phase, the field $\cos \sqrt{2} \phi_{a}$ is disordered and the $V_{2}$ term dominates transport. If $K>1$ this term is irrelevant and the conductance tends at low temperatures to the quantum of conductance. In the vortex phase on the other hand, the field $\cos \sqrt{2} \phi_{a}$ has quasi long range order and the $V_{1}$ term dominates transport, leading (for $K<2$ ) to an insulating state at zero temperature. More generally, even when $K<1$ or $K>2$ the conductance has a qualitatively different temperature dependence in the Meissner and in the vortex phase.

\section{Acknowledgments}

We thank P. Donohue, B. Douçot, A. Georges and J. Vidal for illuminating discussions, and an a referee for pointing out to us Ref. 10,11. This work has been supported in part by the NATO grant No. 971615 .
* Electronic address: orignac@lpt.ens.fr

$\dagger$ Electronic address: giam@lps.u-psud.fn

1 H. J. Mikeska and H. Schmidt, J. Low Temp. Phys 2, 371 (1970).

2 E. Dagotto and T. M. Rice, Science 271, 618 (1996), and references therein.

3 M. Bockrath, D. H. Cobden, J. Lu, A. G. Rinzler, R. E. Smalley, L. Balents, and P. L. Mceuen, Nature 397, 598 (1999).

4 R. Fazio and H. van der Zant, Quantum phase transitions and vortex dynamics in superconducting networks (2000), cond-mat/0011152.

5 A. van Oudenaarden and J. E. Mooij, Phys. Rev. Lett. 76, 4947 (1996).

6 A. van Oudenaarden, S. . J. K. Várdy, and J. Mooij, Phys. Rev. Lett. 77, 4257 (1996).

7 R. M. Bradley and S. Doniach, Phys. Rev. B 30, 1138 (1984).

8 L. Glazman and A. Larkin, Phys. Rev. Lett. 79, 3736 (1997), cond-mat/9705169.

9 C. Denniston and C. Tang, Phys. Rev. Lett. 75, 3930 (1995).

10 M. Kardar, Phys. Rev. B 33, 3125 (1986).

11 E. Granato, Phys. Rev. B 42, 4797 (1990).

12 Y. Nishiyama, Eur. Phys. J. B 17, 295 (2000), cond- mat/0006311.

13 E. Orignac and T. Giamarchi, Phys. Rev. B 57, 11713 (1998).

14 F. D. M. Haldane, Phys. Rev. Lett. 47, 1840 (1981).

15 T. Giamarchi, Physica B 230-232, 975 (1997).

16 T. Giamarchi and A. J. Millis, Phys. Rev. B 46, 9325 (1992).

17 H. J. Schulz, Strongly Correlated Electronic Materials: The Los Alamos Symposium 1993 (Addison-Wesley, Reading, Massachusetts, 1994), p. 187.

18 M. Oshikawa, M. Yamanaka, and I. Affleck, Phys. Rev. Lett. 78, 1984 (1997).

19 D. Cabra, A. Honecker, and P. Pujol, Phys. Rev. Lett. 79, 5126 (1997).

20 G. I. Japaridze and A. A. Nersesyan, JETP Lett. 27, 334 (1978).

21 V. L. Pokrovsky and A. L. Talapov, Phys. Rev. Lett. 42, 65 (1979).

22 H. J. Schulz, Phys. Rev. B 22, 5274 (1980).

23 L. P. Lévy, G. Dolan, J. Dunsmuir, and H. Bouchiat, Phys. Rev. Lett. 64, 2074 (1990).

${ }^{24}$ P. Donohue and T. Giamarchi, Phys. Rev. B 63, 180508(R) (2001). 